\title{
Thrombus imaging in acute stroke: correlation of thrombus length on susceptibility-weighted imaging with endovascular reperfusion success
}

\author{
Christian Weisstanner • Pascal P. Gratz • Gerhard Schroth • Rajeev K. Verma • \\ Arnold Köchl • Simon Jung • Marcel Arnold • Jan Gralla • Christoph Zubler • Kety Hsieh • \\ Pasquale Mordasini • Marwan El-Koussy
}

Received: 16 March 2014 /Revised: 9 April 2014 / Accepted: 23 April 2014 / Published online: 16 May 2014

(C) The Author(s) 2014. This article is published with open access at Springerlink.com

\begin{abstract}
Objectives Susceptibility-weighted imaging (SWI) enables visualization of thrombotic material in acute ischemic stroke. We aimed to validate the accuracy of thrombus depiction on SWI compared to time-of-flight MRA (TOF-MRA), first-pass gadolinium-enhanced MRA (GE-MRA) and digital subtraction angiography (DSA). Furthermore, we analysed the impact of thrombus length on reperfusion success with endovascular therapy.

Methods Consecutive patients with acute ischemic stroke due to middle cerebral artery (MCA) occlusions undergoing endovascular recanalization were screened. Only patients with a pretreatment SWI were included. Thrombus visibility and location on SWI were compared to those on TOF-MRA, GE-MRA and DSA. The association between thrombus length on SWI and reperfusion success was studied.

Results Eighty-four of the 88 patients included (95.5\%) showed an MCA thrombus on SWI. Strong correlations between thrombus location on SWI and that on TOF-MRA (Pearson's correlation coefficient 0.918, $P<0.001$ ), GE-MRA $(0.887, P<0.001)$ and DSA $(0.841, P<0.001)$ were observed.
\end{abstract}

C. Weisstanner • P. P. Gratz · G. Schroth $(\bowtie) \cdot$ R. K. Verma

A. Köchl $\cdot$ S. Jung $\cdot$ J. Gralla $\cdot$ C. Zubler $\cdot$ K. Hsieh $\cdot$ P. Mordasini $\cdot$ M. El-Koussy

Department of Diagnostic and Interventional Neuroradiology, Inselspital, Bern University Hospital and University of Bern, Bern, Switzerland

e-mail: gerhard.schroth@insel.ch

S. Jung $\cdot$ M. Arnold

Department of Neurology, Inselspital, Bern University Hospital and University of Bern, Bern, Switzerland
Successful reperfusion was not significantly related to thrombus length on SWI ( $P=0.153$; binary logistic regression).

Conclusions In MCA occlusion thrombus location as seen on SWI correlates well with angiographic findings. In contrast to intravenous thrombolysis, thrombus length appears to have no impact on reperfusion success of endovascular therapy.

Key Points

- SWI helps in assessing location and length of thrombi in the $M C A$

- SWI, MRA and DSA are equivalent in detecting the MCA occlusion site

- SWI is superior in identifying the distal end of the thrombus

- Stent retrievers should be deployed over the distal thrombus end

- Thrombus length did not affect success of endovascular reperfusion guided by $S W I$

Keywords Acute stroke · Intracranial thrombus · Magnetic resonance imaging · Digital subtraction angiography ·

Thrombectomy

Abbreviations

DSA Digital subtraction angiography

GE-MRA Gadolinium-enhanced magnetic resonance angiography

iv-tPA Intravenous recombinant tissue plasminogen activator

MCA Middle cerebral artery

mIP Minimum intensity projection

mRS Modified Rankin scale

NIHSS National Institutes of Health stroke scale

SVS Susceptibility vessel sign 
SWI Susceptibility-weighted imaging

TICI Thrombolysis in cerebral infarction

TOF-MRA Time-of-flight magnetic resonance angiography

\section{Introduction}

Improved functional outcome following acute ischemic stroke is strongly correlated with successful vessel recanalization [1]. A study on middle cerebral artery (MCA) stroke demonstrated that intravenous thrombolysis had nearly no potential for recanalizing thrombi measuring more than $8 \mathrm{~mm}$ in length [2].

Susceptibility-weighted imaging (SWI), a fully velocitycompensated high-resolution 3D gradient-echo sequence, is becoming an essential technique in the primary and follow-up MRI evaluation of patients with cerebrovascular disease [3]. SWI helps in distinguishing structures that have different susceptibility values than their surroundings [4]. Applications of SWI in stroke imaging include the exclusion of intracranial haemorrhage before administration of intravenous thrombolytics, visualization of prominent cortical veins that have been shown to indicate cerebral misery perfusion [5] and detection of haemorrhagic infarct transformation. An additional application of SWI is the direct visualization of thrombotic material [6]. A recent study has shown an excellent correlation between thrombus length as measured on $\mathrm{T} 2 *$ gradient-echo sequences compared to that determined by digital subtraction angiography (DSA) in patients with acute anterior and posterior circulation stroke [7].

The aim of this study was first to analyse the accuracy of SWI in visualizing the occlusion site compared to traditional MRI angiographic sequences as well as DSA and second, to investigate whether thrombus length as measured on SWI affects the success of endovascular reperfusion therapy of MCA occlusions.

\section{Patients and methods}

A total of 396 consecutive acute ischemic stroke patients treated by endovascular means at our stroke unit, from March 2010 to May 2012, were screened. Inclusion criteria were isolated MCA M1 and/or M2 segment occlusion, preinterventional MRI including SWI, and endovascular reperfusion therapy. A total of 88 patients fulfilled the inclusion criteria. The study was approved by our institutional review board. All patients gave written informed consent prior to enrolment in our stroke registry. If patients did not have the capacity to consent, written informed consent was obtained from their nearest relatives.
Clinical data on patients with acute ischemic stroke were prospectively recorded in our stroke database. Neurologic deficits were scored by a neurologist using the National Institutes of Health stroke scale (NIHSS). Clinical outcome was assessed 3 months after stroke using the modified Rankin scale (mRS).

Image acquisition

Standard stroke MRI protocol was performed, which included diffusion-weighted imaging, T2-weighted imaging, time-offlight magnetic resonance angiography (TOF-MRA), SWI, perfusion imaging, 3D first-pass gadolinium-enhanced MRA (GE-MRA) of the cervical and intracranial arteries, and T1weighted post-contrast imaging. The images were acquired with a 1.5-T and 3-T MRI (Magnetom Avanto and Magnetom Verio; Siemens, Erlangen, Germany).

For the 1.5-T device, the SWI parameters were TR $49 \mathrm{~ms}$, TE $40 \mathrm{~ms}$, number of averages 1 , FoV read $230 \mathrm{~mm}$, FoV phase $75.0 \%$, voxel size $0.9 \times 0.7 \times 1.8 \mathrm{~mm}$, flip angle $15^{\circ}$, acquisition time $2: 59 \mathrm{~min}$. For the 3 -T device the parameters were as follows: TR $28 \mathrm{~ms}$, TE $20 \mathrm{~ms}$, number of averages 1 , FoV read $230 \mathrm{~mm}$, FoV phase $75.0 \%$, voxel size $0.9 \times 0.9 \times$ $2.0 \mathrm{~mm}$, flip angle $15^{\circ}$, acquisition time 2:59 $\mathrm{min}$. The SWI and minimum intensity projection (mIP) images were generated automatically by the scanner software.

The TOF-MRA parameters were as follows: 36 slices per slab, 4 slabs, $0.60 \mathrm{~mm}$ thickness, TR $22 \mathrm{~ms}$, TE $3.6 \mathrm{~ms}$, number of averages 1 , FoV read $200 \mathrm{~mm}$, FoV phase $90.6 \%$, voxel size $0.5 \times 0.5 \times 0.6 \mathrm{~mm}$, flip angle $18^{\circ}$, acquisition time 5:16 $\mathrm{min}$.

For the GE-MRA of the cranial and cervical arteries 88 coronal slices were acquired with $0.9 \mathrm{~mm}$ thickness, TR $3.21 \mathrm{~ms}$, TE $1.2 \mathrm{~ms}$, number of averages 1 , FoV read $300 \mathrm{~mm}$, FoV phase $100 \%$, voxel size $1.0 \times 0.8 \times 0.9 \mathrm{~mm}$, flip angle $25^{\circ}, 4$ measurements, acquisition time 1:30 min.

DSA was performed via a transfemoral approach using a biplane, high-resolution angiographic system (Axiom Artis zee; Siemens, Erlangen, Germany).

Imaging analysis

Pre-interventional SWI and mIP series were analysed for the presence of a susceptibility vessel sign (SVS) in the MCA by three radiologists (C.W., R.K.V., A.K.). SVS was defined as an area of signal drop within the course of an artery that either exceeded the diameter of the contralateral non-occluded vessel, or that of the adjacent vessel segment on both the SWI and mIP series [8]. Discrepancies between reviewers regarding thrombus visibility on SWI were resolved in consensus. After analysis of the SWI the presence of a vessel occlusion on TOF-MRA and GE-MRA corresponding to the proximal thrombus end on SWI was determined. 
The reviewers were aware of the presence of an MCA occlusion, but were blinded to lesion side and clinical symptoms.

Next, maximum thrombus length was measured on SWI using a previously published method [7]. The location of the SVS was determined. The position of the proximal thrombus end was defined in relation to the midline (Figs. 1 and 2). The midline was defined as a line connecting the occipital part of the superior sagittal sinus with the point midway between the A2 segments of the anterior cerebral arteries as seen on the axial images. TOF-MRA, GE-MRA and diagnostic series of the DSA were analysed for the presence and location of the occluding thrombus according to the same criteria as on SWI. On the coronal GE-MRA (Fig. 2c) and the anteroposterior DSA projections (Fig. 2d), the midline was defined as a perpendicular line, midway between the A2 segments. Measurements were done by a radiologist in training (P.P.G.), who was aware of the presence of an SVS on SWI and the location of the occlusion on TOF-MRA, GEMRA and DSA.

The following endovascular recanalization techniques were applied: stent retriever thrombectomy, thromboaspiration, extra- and intracranial stenting, intra-arterial thrombolysis or

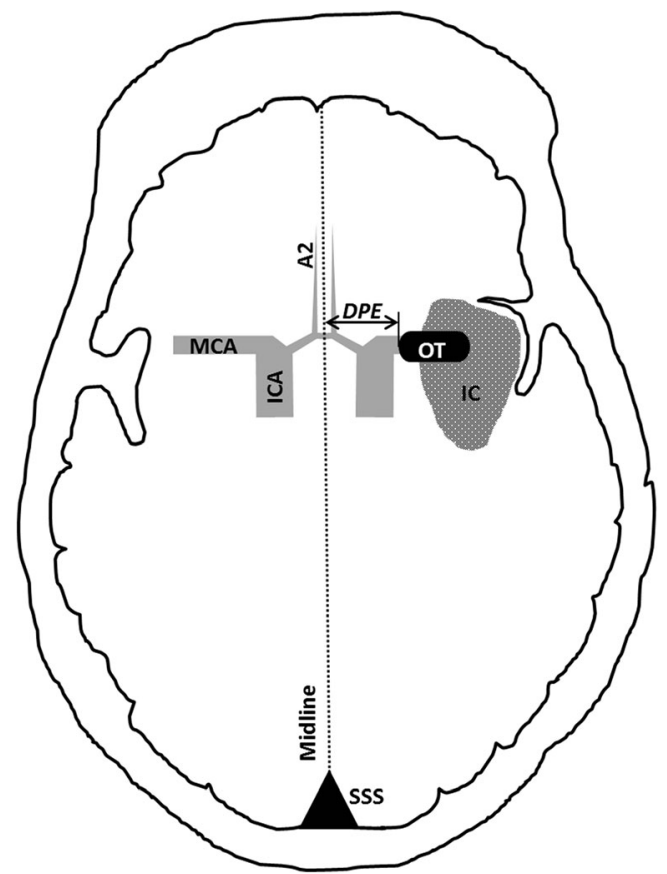

Fig. 1 Measurement of thrombus localization. Midline was defined as a line connecting the occipital part of the superior sagittal sinus with the point midway between the A2 segments of the anterior cerebral arteries as seen on axial SWI and TOF-MRA. On coronal GE-MRA and anteroposterior DSA projections, midline was defined as a perpendicular line, midway between the A2 segments (OT occluding thrombus, IC infarct core, DPE distance to proximal end of thrombus, SSS superior sagittal sinus, ICA internal carotid artery, $M C A$ middle cerebral artery, $A 2$ A2 segment of the anterior cerebral artery)
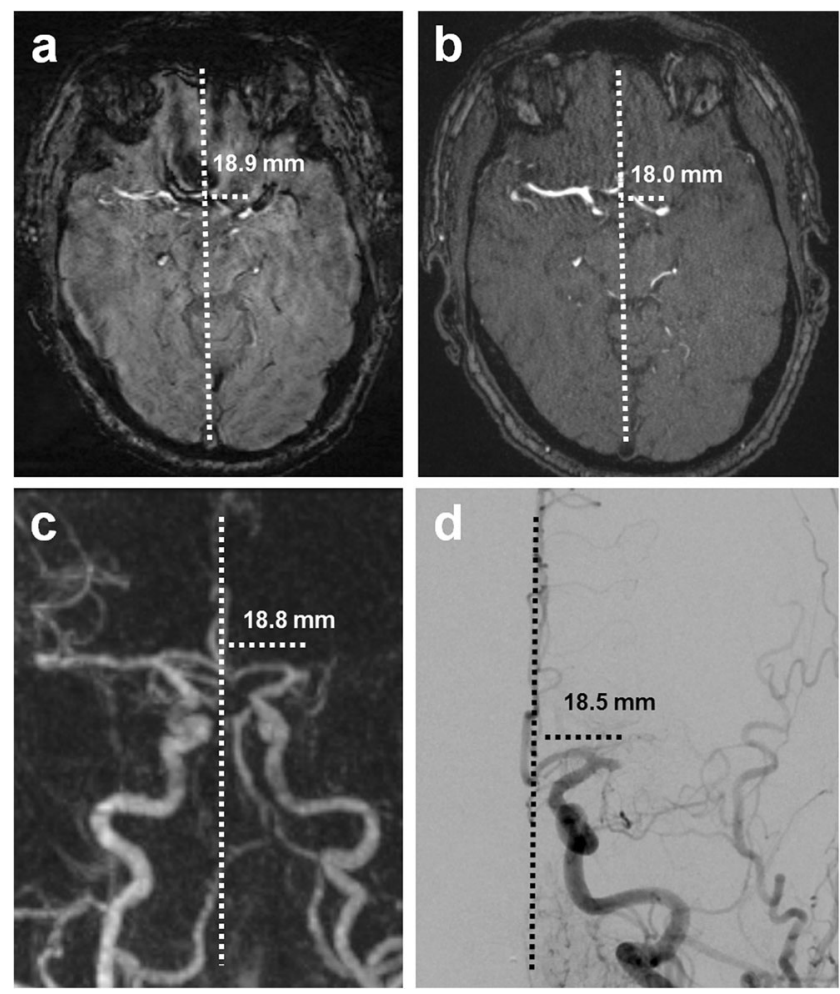

Fig. 2 60-year-old man with global aphasia and right-sided hemiparesis (NIHSS score 17). a On SWI a thrombus is visible in the left MCA. The distance of the proximal thrombus end to the midline on SWI is within the 1-mm range compared to that measured on $\mathbf{b}$ TOF-MRA, $\mathbf{c}$ GE-MRA and d DSA

any combination of these. The interventional team selected material and thrombectomy techniques on the basis of the thrombus extension and angioarchitecture of the arteries proximal and distal to the occlusion site as seen on multimodal stroke MRI protocol. Stent retrievers were always deployed with the tip distal to the thrombus end. Cerebral reperfusion was assessed at the end of the endovascular intervention, on biplane or 3D rotational angiography, according to the thrombolysis in cerebral infarction (TICI) grading system [9].

\section{Statistical analysis}

Continuous variables are presented as mean \pm standard deviation (SD) if not stated otherwise. Successful reperfusion was defined as TICI grades $2 b-3$. Pearson correlation coefficients were used to compare thrombus location (distance of proximal thrombus end to midline) between the different imaging modalities (i.e. SWI, TOF-MRA, GE-MRA and DSA). BlandAltman plots were used to identify systematic differences between measurements of thrombus location on SWI and the other imaging modalities [10]. Binary logistic regression was used to compare thrombus length on SWI with reperfusion success. The correlation between thrombus length on SWI and NIHSS score on admission was studied by Kendall's $\tau$ rank correlation test. Associations between thrombus 
location and NIHSS score on admission were analysed with the Kruskal-Wallis test. A $P$ value less than 0.05 was considered significant. Interobserver reliability for the detection of the SVS was studied using Cohen's K statistics. Statistics were performed with SPSS version 21 (IBM Corp., Armonk, NY, USA) and R version 3.0.3 (R Foundation for Statistical Computing, Vienna, Austria).

\section{Results}

A total of 46 women and 42 men were studied. Their mean age was $66.5 \pm 15.9$ years (range 13-89 years).

Imaging of occluding thrombi

A total of 69 patients had an occlusion of the M1, 17 of the M2 and two of both the M1 and M2 segments. Interobserver reliability for the detection of SVS on SWI was fair to excellent and ranged from 0.535 to 0.788 between pairs of reviewers (Cohen's K). After consensus review, an SVS corresponding to the occluding thrombus was found in 84 of the 88 patients $(95.5 \%)$. TOF-MRA detected a vessel occlusion in 87 patients $(98.9 \%)$. In 79 of 81 patients $(97.5 \%)$ in whom a GE-MRA was performed, a vessel occlusion was identified. In DSA, which served as the gold standard in our study, a vessel occlusion was found in all patients. In the 84 patients with SVS, mean thrombus length on SWI was $10.9 \pm 4.7 \mathrm{~mm}$ (range $4.6-30.5 \mathrm{~mm}$ ).

The distances of the proximal thrombus end to the midline were $26.4 \pm 6.3 \mathrm{~mm}$ for SWI, $24.8 \pm 6.1 \mathrm{~mm}$ for TOF-MRA, 27.4 $\pm 6.1 \mathrm{~mm}$ for GE-MRA and $26.6 \pm 6.4 \mathrm{~mm}$ for DSA. Strong correlations were found between the location of the proximal thrombus end on SWI and that on TOF-MRA (Pearson's correlation coefficient $0.918, P<0.001$ ), GE-MRA (Pearson's correlation coefficient $0.887, P<0.001$ ) and DSA (Pearson's correlation coefficient $0.841, P<0.001)$. The Bland-Altman plots showed a mean bias of $1.6 \mathrm{~mm}$ (precision $2.5 \mathrm{~mm}$ [SD of bias]; percentage error $6.4 \%$ [precision divided by mean distance of proximal thrombus end to midline]) between SWI and TOFMRA, of $-1.0 \mathrm{~mm}$ (precision $3.0 \mathrm{~mm}$; percentage error $-3.7 \%$ ) between SWI and GE-MRA, and $-0.1 \mathrm{~mm}$ (precision $3.8 \mathrm{~mm}$; percentage error $-0.3 \%$ ) between SWI and DSA (Fig. 3).

\section{Treatment}

Table 1 gives an overview of clinical characteristics, treatment, reperfusion success and clinical outcome. A total of 28 patients $(31.8 \%)$ received intravenous thrombolysis with recombinant tissue-type plasminogen activator (iv-tPA) before endovascular treatment. Median tPA dose was $47 \mathrm{mg}$ (range $31-72 \mathrm{mg}$ ). In 25 patients iv-tPA was started directly after acquiring the MRI. In these patients the mean time from symptom onset to MRI acquisition was $125.7 \pm 40.9 \mathrm{~min}$. In
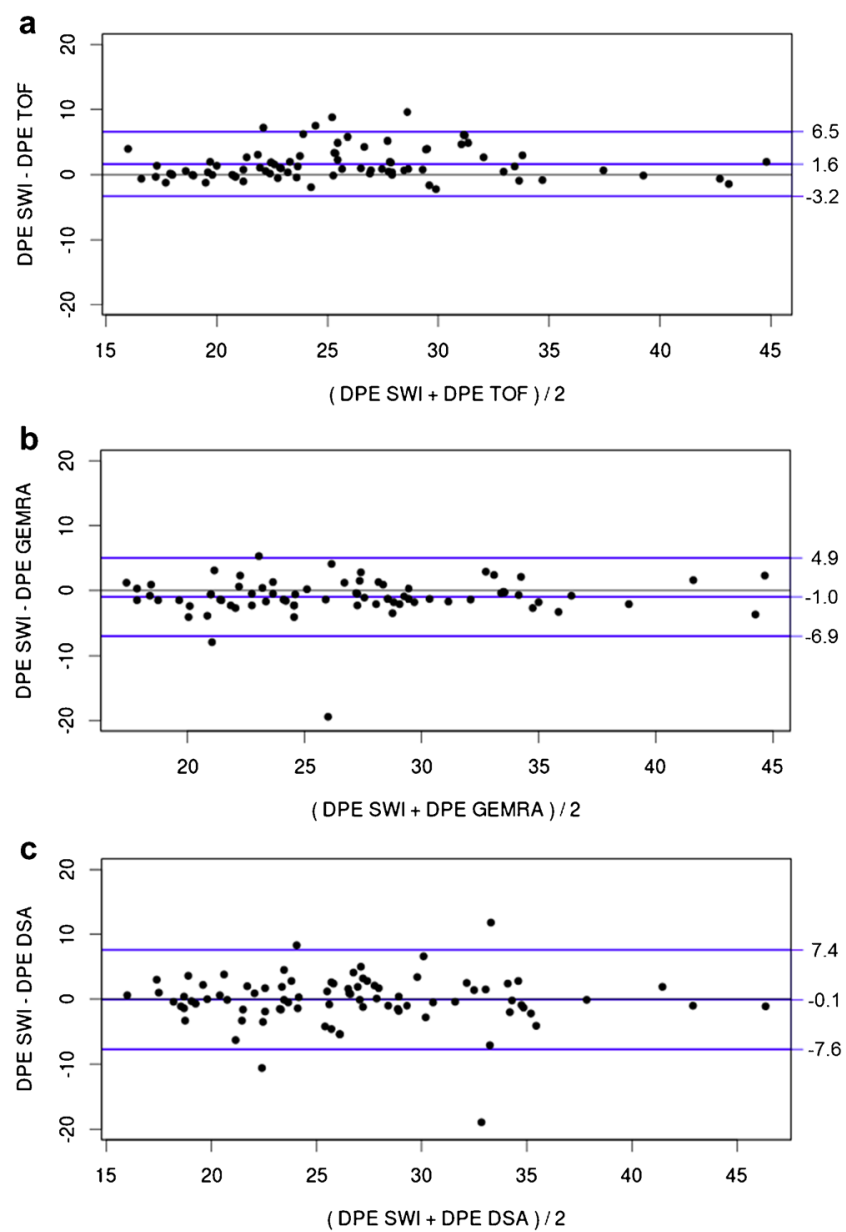

Fig. 3 Bland-Altman plots for measurements of distance of proximal thrombus end to midline (DPE) on SWI and corresponding measurements on TOF-MRA (a), GE-MRA (b) and DSA (c) showing mean bias and upper and lower limits of agreement (mean bias $\pm 1.96 \times$ standard deviations). Units are in millimetres

three patients, iv-tPA was administered on the way to our institution after acquisition of a head $\mathrm{CT}$ and exclusion of contraindications for intravenous thrombolysis at an outside hospital. In these cases, MRI was performed for further treatment planning on admission of the patient at our hospital.

Most patients underwent multimodal endovascular therapy and in the majority, stent retriever thrombectomy was performed.

Association of thrombus length and location with clinical parameters and reperfusion success

A weak correlation was found between thrombus length on SWI and baseline NIHSS score (Kendall's $\tau$ coefficient 0.197; $P=0.010$ ). When analysing the association between occlusion site and baseline NIHSS score the Kruskal-Wallis test demonstrated a non-significant trend $(P=0.055)$. Patients with M1 or a combination of M1 and M2 occlusions had higher median NIHSS scores on admission compared to patients with isolated M2 occlusions (NIHSS score 15 versus 9). 
Table 1 Imaging, clinical and procedural characteristics in 88 patients with middle cerebral artery occlusion

$\begin{array}{lr}\text { Time from symptom onset to MRI, min, mean (SD) } & 181.1(112.4) \\ \text { Time from symptom onset to DSA, min, mean (SD) } & 250.0(118.1) \\ \text { Baseline NIHSS score, median (range) } & 13.5(3-36) \\ \text { Stroke aetiology } & \\ \quad \text { Large artery disease, } n(\%) & 5(5.7) \\ \quad \text { Cardioembolic, } n(\%) & 41(46.6) \\ \quad \text { Other determined aetiology, } n(\%) & 11(12.5) \\ \quad \text { Unknown aetiology, } n(\%) & 31(35.2) \\ \text { Intravenous bridging thrombolysis, } n(\%) & 28(31.8) \\ \text { Successful reperfusion (TICI 2b-3), } n(\%) & 62(70.5) \\ \text { Interventional characteristics, } n(\%) & \\ \quad \text { Stent retriever thrombectomy } & 67(76.1) \\ \quad \text { Thromboaspiration } & 4(4.5) \\ \quad \text { Extra- and intracranial stenting } & 9(10.1) \\ \quad \text { Intra-arterial thrombolysis } & 35(39.8) \\ \text { Favourable outcome (mRS, } 0-2) \text { at } 3 \text { months, } n(\%) & 40 / 85(47.1) \\ \text { Dead at } 3 \text { months, } n \text { (\%) } & 14 / 86(16.3)\end{array}$

NIHSS National Institutes of Health stroke scale, TICI thrombolysis in cerebral infarction, $m R S$ modified Rankin scale.

Successful reperfusion (TICI $2 \mathrm{~b}-3)$ was achieved independent of thrombus length on SWI $(P=0.153$; OR $0.924 ; 95 \%$ CI, 0.830-1.030) using multimodal endovascular technique.

\section{Discussion}

In our series of patients with MCA occlusions, $95.5 \%$ of the thrombi were clearly definable on SWI. This detection rate is in line with those recently reported for SWI and T2* gradientecho imaging in anterior circulation stroke $[7,11]$. We propose a new technique for defining thrombus location that is applicable to different imaging modalities (Fig. 1). Using this technique we found a strong correlation between the location of the proximal thrombus end as seen on SWI and that on TOF-MRA, GE-MRA and DSA. These findings support previous reports which showed that SWI and T2* gradient-echo imaging can reliably depict thrombus location when compared to TOF-MRA [11] and DSA [7]. Moreover, we demonstrated that thrombus length as measured on SWI did not appear to have an impact on success of endovascular reperfusion therapy of MCA occlusions.

The latter finding is of particular interest. Various studies have shown that the success of vessel recanalization with intravenous thrombolysis seems to depend on clot burden. Clot burden was defined either on thin-slice non-enhanced CT, CT-angiography, or recently on $\mathrm{T} 2 *$ gradient-echo images measuring either absolute thrombus length [2] or applying a scoring system based on thrombus location $[12,13]$. MCA thrombi measuring more than $8 \mathrm{~mm}$ in length were found to have nearly no potential for recanalization with intravenous thrombolysis [2]. Lower clot burden scores, indicating a higher thrombus burden, showed significant associations with worse clinical outcome $[12,13]$.

The effect of thrombus burden on reperfusion success and clinical outcome after endovascular treatment remains less clear. A study by Puetz et al. analysing clinical outcome in relation to the clot burden score on CT-angiography found no differential effect on outcome between patients treated by intravenous thrombolysis, endovascular means or a combination of both [12]. Barreto et al. used the modified TIMI criteria to assess thrombus burden in patients undergoing endovascular therapy alone or in combination with bridging thrombolysis [14]. In his study, a significant association between high clot burden and worse clinical outcome at 3 months was observed. Although the percentage of patients with successful reperfusion (TICI 2b-3) was higher in the group with lower clot burden compared to those with larger clots, this difference was not significant [14]. In the present study, thrombus length as measured on SWI did not significantly affect reperfusion success $(P=0.153)$. Our findings suggest that when using multimodal endovascular revascularization techniques, thrombus length does not affect the chances of successful reperfusion. It has to be noted that the comparability between the aforementioned studies is limited because of the variable methods used to assess clot burden or size.

Detection rates of vessel occlusions were similar between SWI (95.5 \%), TOF-MRA (98.9 \%) and GE-MRA (97.5\%). In two of the four patients with no definable SVS, the interpretability of SWI was limited because of severe motion artefacts and in one patient with a proximal M1 occlusion, the MCA was partially obscured by susceptibility artefacts in the proximity of the skull base. In the fourth patient the thrombus could not be detected on SWI for unknown reasons, even in direct comparison with TOF-MRA, GE-MRA and DSA which clearly depicted a vessel occlusion.

Interestingly, small but systematic differences were found between the distances of the proximal thrombus end to the midline measured on SWI and those obtained on TOF-MRA (mean bias $1.6 \mathrm{~mm}$; percentage error $6.4 \%$ ) using BlandAltman statistics (Fig. 3). On TOF-MRA slow flow, such as seen proximal to an occlusion, can lead to inadequate depiction of vessel segments that are still patent, potentially resulting in overestimation of the occlusion $[15,16]$. This phenomenon might explain the shorter distance measured from midline to proximal thrombus end on TOF-MRA compared with the other angiographic methods.

Although TOF-MRA and GE-MRA remain indispensable in stroke imaging, SWI adds important complementary information. SWI not only shows the proximal end of the occluding thrombus, but also its length. For these reasons SWI has become a valuable part of our stroke imaging protocol. For optimal planning of the endovascular procedure, we deem it 
necessary to know the thrombus extension as well as each individual patient's angioarchitecture from the aortic arch up to the distal branches of the large intracranial arteries. In daily practice thrombus imaging with SWI guides us in the selection of the treatment approach in patients with MCA occlusions. In patients with relatively short thrombi measuring less than $8 \mathrm{~mm}$ in length we preferentially use intravenous thrombolysis and continue with endovascular therapy only in case the symptoms do not improve [2]. In patients with longer thrombi limited to the sphenoidal segment of the MCA (M1 segment), stent retriever thrombectomy is the treatment of choice. In the absence of contraindications, bridging thrombolysis is usually initiated before endovascular treatment. When using stent retrievers it is important to deploy the distal tip of the device in the vessel segment distal to the thrombus so as not to shear off parts of the clot during stent retraction. Again, SWI is helpful for planning this procedure as it can identify the distal thrombus end as well as the curvature of the occluded vessel segment. This information is especially important in patients with thrombi extending from the M1 segment to the insular segment (M2) or even affecting opercular branches (M3). In order to deploy a stent retriever, the microcatheter has first to be navigated without the aid of a roadmap, through the curved vasculature at the transition of the sphenoidal to the opercular segment, or even through the vessels in the limen insulae. Furthermore, diameter, length and type of the stent retriever can be selected prior to the intervention on the basis of information from the SWI. Additional techniques, such as the use of distal access aspiration catheters [17], can be prearranged and applied in complex thrombus and vessel anatomy. Figure 4 shows different types of thrombi as seen on SWI warranting different treatment approaches.

Our study has some limitations. One could argue that it is unknown to what extent the distal end of the susceptibility vessel sign is caused by the thrombus itself, and to what extent by stagnating, deoxyhaemoglobin-rich blood distal to the occlusion, resulting in overestimation of the real thrombus length. A recent study addressed this uncertainty by showing an excellent correlation between the thrombus length as measured on T2* gradient-echo imaging and that on DSA [7]. To avoid the limited sensitivity of SWI in proximity to the skull base, i.e. intracranial segment of the internal carotid artery and posterior circulation arteries, we decided to assess only MCA occlusions to correlate the sensitivity of SWI with various angiographic techniques. The sensitivity of gradient-echo imaging in detecting thrombi appears to be lower for the posterior circulation [7]. Only few patients presented with isolated occlusions of the M2 segment and none had an occlusion of the M3/4 segments. The generalizability of the results regarding the thrombus location defined by vessel segment is therefore limited because of the rather small patient number. Furthermore, all patients included in our study underwent endovascular treatment. Such patients usually present with
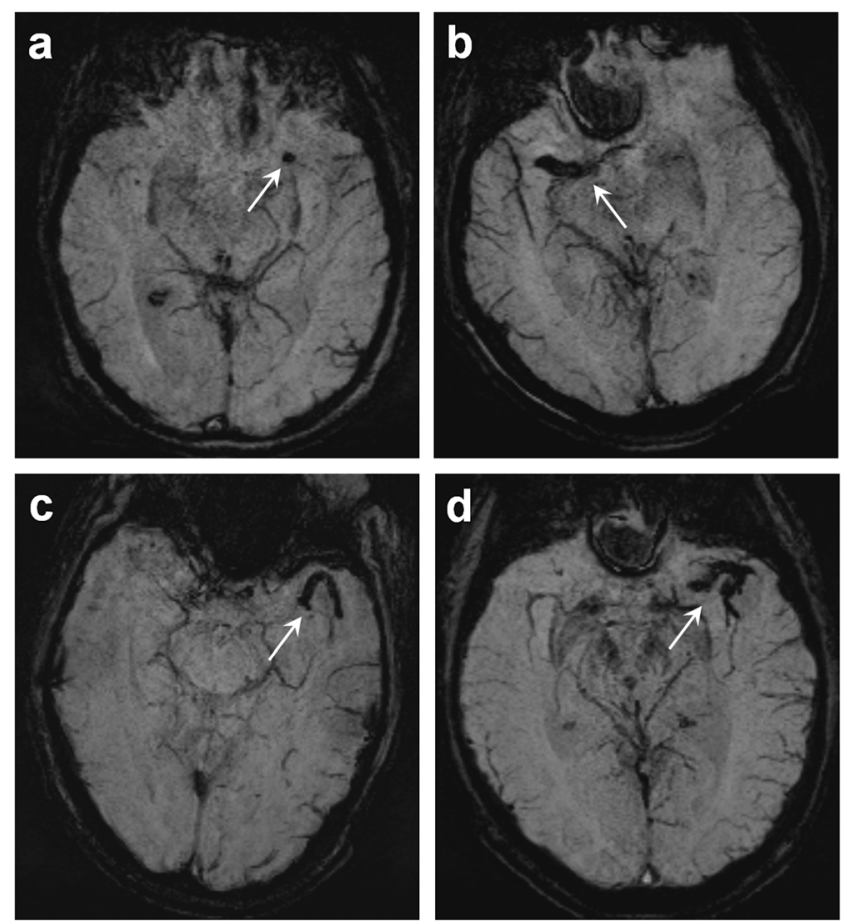

Fig. 4 Evaluation of thrombus anatomy (white arrows) on SWI (mIP). Patients with thrombi measuring less than $8 \mathrm{~mm}$ in length are preferentially treated with intravenous thrombolysis (a). Thrombi limited to the M1 segment but measuring more than $8 \mathrm{~mm}$ can usually be removed easily by stent retriever thrombectomy (b). Treatment of thrombotic occlusions extending from the M1 segment to one (c) or multiple (d) distal branches is generally more challenging. If the interventionist decides to deploy a stent retriever first a microcatheter has to be navigated blindly through the occluded vessel. For planning of this manoeuvre, SWI proves to be helpful as it visualizes the curvature of the occluded vessel segment

more proximal and larger thrombi compared to those treated by intravenous thrombolysis alone. Thrombus length has been shown to influence detectability of thrombi on $\mathrm{T} 2 *$ gradientecho imaging [7]. An additional bias may have been introduced by obtaining SWI at two different field strengths (1.5 T and $3 \mathrm{~T}$ ). A study analysing the effect of field strength on the diameter of cerebral microbleeds as measured on SWI showed an increase in size with higher field strength [18]. Likewise, the length of the SVS on SWI may slightly vary with field strength. Finally, SWI tends to overestimate the real proportions of material with different magnetic susceptibility than the surrounding tissue (e.g. microbleeds, thrombi) [19, 20], probably resulting in a slight overestimation of maximal thrombus length.

\section{Conclusions}

This study demonstrates that SWI is a valuable addition to the standard stroke MRI protocol. Thrombus location on SWI correlated strongly with that on TOF-MRA, GE-MRA and DSA. Reperfusion success of multimodal endovascular 
treatment techniques seems not to be affected by thrombus length as measured on SWI if information on thrombus extension with regard to the individual patient's angioarchitecture is included in endovascular treatment planning.

Acknowledgments The scientific guarantor of this publication is Prof. Dr. Gerhard Schroth. The authors of this manuscript declare no relationships with any companies whose products or services may be related to the subject matter of the article. The authors state that this work has not received any funding. Pietro Ballinari and Martin Zbinden kindly provided statistical advice for this manuscript. Institutional review board approval was obtained. Written informed consent was obtained from all patients, or in case patients did not have the capacity to consent from their nearest relatives, in this retrospective, diagnostic study performed at one institution. The authors appreciate the support of the MRI technologists, headed by Carole Stuker, and angiography technologists, headed by Thierry Horisberger, at our institute.

Christian Weisstanner and Pascal P. Gratz contributed equally to this study.

Open Access This article is distributed under the terms of the Creative Commons Attribution Noncommercial License which permits any noncommercial use, distribution, and reproduction in any medium, provided the original author(s) and the source are credited.

\section{References}

1. Rha JH, Saver JL (2007) The impact of recanalization on ischemic stroke outcome: a meta-analysis. Stroke 38:967-973

2. Riedel CH, Zimmermann P, Jensen-Kondering U, Stingele R, Deuschl G, Jansen O (2011) The importance of size: successful recanalization by intravenous thrombolysis in acute anterior stroke depends on thrombus length. Stroke 42:1775-1777

3. Huang P, Chen CH, Lin WC et al (2012) Clinical applications of susceptibility weighted imaging in patients with major stroke. J Neurol 259:1426-1432

4. Haacke EM, Mittal S, Wu Z, Neelavalli J, Cheng YC (2009) Susceptibility-weighted imaging: technical aspects and clinical applications, part 1. AJNR Am J Neuroradiol 30:19-30

5. Kao HW, Tsai FY, Hasso AN (2012) Predicting stroke evolution: comparison of susceptibility-weighted MR imaging with MR perfusion. Eur Radiol 22:1397-1403
6. Mittal S, Wu Z, Neelavalli J, Haacke EM (2009) Susceptibilityweighted imaging: technical aspects and clinical applications, part 2. AJNR Am J Neuroradiol 30:232-252

7. Naggara O, Raymond J, Domingo Ayllon M et al (2013) T2* "susceptibility vessel sign" demonstrates clot location and length in acute ischemic stroke. PLoS ONE 8:e76727

8. Rovira A, Orellana P, Alvarez-Sabín J et al (2004) Hyperacute ischemic stroke: middle cerebral artery susceptibility sign at echoplanar gradient-echo MR imaging. Radiology 232:466-473

9. Higashida RT, Furlan AJ, Roberts H et al (2003) Trial design and reporting standards for intra-arterial cerebral thrombolysis for acute ischemic stroke. Stroke 34:e109-e137

10. Bland JM, Altman DG (1986) Statistical methods for assessing agreement between two methods of clinical measurement. Lancet $1: 307-310$

11. Radbruch A, Mucke J, Schweser F et al (2013) Comparison of susceptibility weighted imaging and TOF-angiography for the detection of thrombi in acute stroke. PLoS ONE 8:e63459

12. Puetz V, Dzialowski I, Hill MD et al (2008) Intracranial thrombus extent predicts clinical outcome, final infarct size and hemorrhagic transformation in ischemic stroke: the clot burden score. Int J Stroke 3:230-236

13. Legrand L, Naggara O, Turc G et al (2013) Clot burden score on admission T2*-MRI predicts recanalization in acute stroke. Stroke 44:1878-1884

14. Barreto AD, Albright KC, Hallevi $\mathrm{H}$ et al (2008) Thrombus burden is associated with clinical outcome after intra-arterial therapy for acute ischemic stroke. Stroke 39:3231-3235

15. Axel L (1986) Blood flow effects in magnetic resonance imaging. Magn Reson Annu 237-244

16. Yang JJ, Hill MD, Morrish WF et al (2002) Comparison of pre- and postcontrast 3D time-of-flight MR angiography for the evaluation of distal intracranial branch occlusions in acute ischemic stroke. AJNR Am J Neuroradiol 23:557-567

17. Gratz PP, Jung S, Schroth G et al (2014) Outcome of standard and high-risk patients with acute anterior circulation stroke after stent retriever thrombectomy. Stroke 45:152-158

18. Nandigam RNK, Viswanathan A, Delgado P et al (2009) MR imaging detection of cerebral microbleeds: effect of susceptibilityweighted imaging, section thickness, and field strength. AJNR Am J Neuroradiol 30:338-343

19. Schrag M, McAuley G, Pomakian J et al (2010) Correlation of hypointensities in susceptibility-weighted images to tissue histology in dementia patients with cerebral amyloid angiopathy: a postmortem MRI study. Acta Neuropathol 119:291-302

20. Greenberg SM, Vernooij MW, Cordonnier C et al (2009) Cerebral microbleeds: a guide to detection and interpretation. Lancet Neurol 8: $165-174$ 